\title{
A Comparison of Simplified Two-dimensional Flow Models Exemplified by Water Flow in a Cavern
}

\author{
Dzmitry Prybytak, Piotr Zima \\ Gdańsk University of Technology, Faculty of Civil and Environmental Engineering, ul. Narutowicza 11/12, \\ 80-952 Gdańsk, Poland, e-mails: dzmpryby@ student.pg.edu.pl, pzim@pg.gda.pl
}

(Received September 6, 2016; revised November 30, 2017)

\begin{abstract}
The paper shows the results of a comparison of simplified models describing a two-dimensional water flow in the example of a water flow through a straight channel sector with a cavern. The following models were tested: the two-dimensional potential flow model, the Stokes model and the Navier-Stokes model. In order to solve the first two, the boundary element method was employed, whereas to solve the Navier-Stokes equations, the open-source code library OpenFOAM was applied. The results of numerical solutions were compared with the results of measurements carried out on a test stand in a hydraulic laboratory. The measurements were taken with an ADV probe (Acoustic Doppler Velocimeter). Finally, differences between the results obtained from the mathematical models and the results of laboratory measurements were analysed.
\end{abstract}

Key words: potential flow, Stokes flow, Navier-Stokes flow, BEM, OpenFOAM

\section{List of symbols}

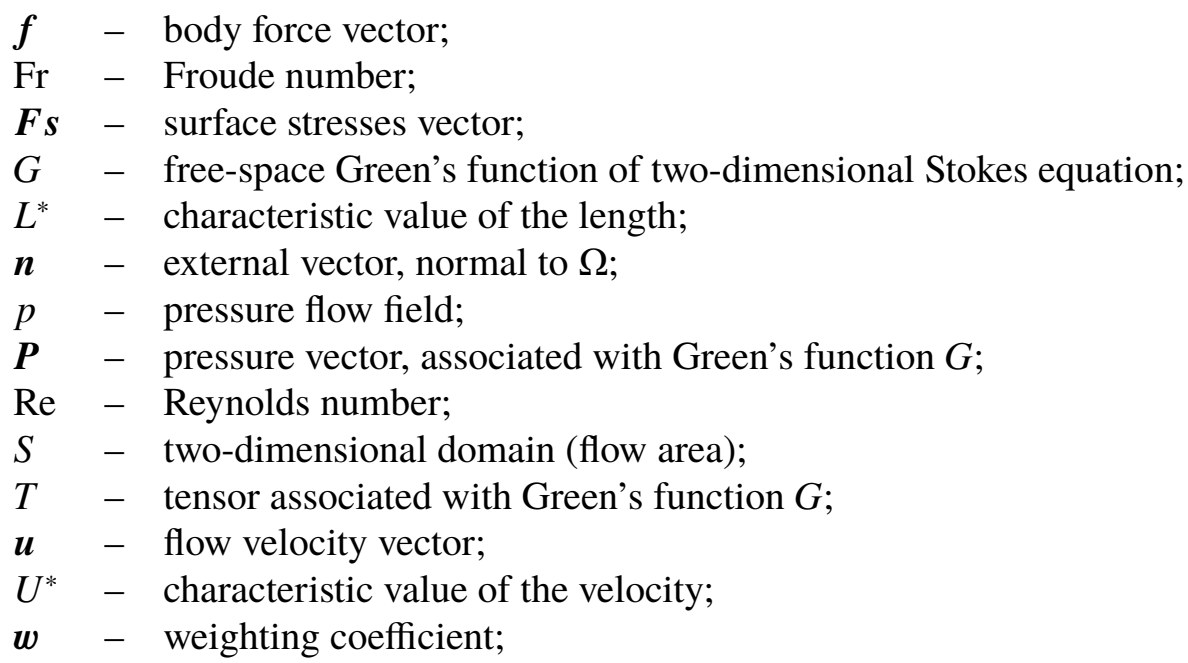




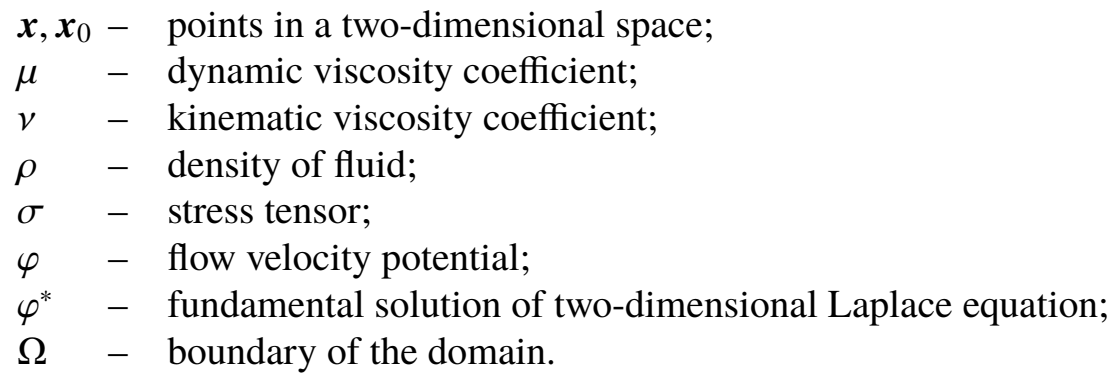

\section{Introduction}

According to the methodology usually applied in building a mathematical model (Meerschaert 2007), one important aspect is the selection of an adequate mathematical description of the phenomenon examined. In a general meaning, such a model is a reasoned object developed to imitate a set of chosen characteristics of another object, which is called the original (Andrews and McLone 1976). This means that a certain mathematical abstraction constitutes a model of a real object in terms of a specific set of characteristics (qualities) of this object. Knowledge about the qualities of the object allows us to define its level of sensitivity (susceptibility) to relevant forces. Most often, simplifying physical assumptions are made to omit the influence of those forces which generate small reactions of the real object. However, the analysis of the level of impact of a given force in each specific case may be individual and based on an agreed modelling objective.

In the case of water flow, the mathematical models applied are created on the basis of mathematical physics equations, which act as a mathematical expression of the laws of conservation and are formulated in the form of problems described by differential equations. These equations usually provide accurate (analytical) solutions only in specific cases, which makes them almost useless for solving technical problems (Landau and Lifshitz 1959). Therefore, in order to solve such problems, various simplified methods are almost always applied, including numerical methods (which are chosen most frequently). This relates to the process of domain discretization and to the adoption of a specific numerical solving method for this kind of equations. Simplifications made in describing a phenomenon concern both its spatial characteristics and the consideration of specific impacts (forces) influencing its course. The more detailed a model is, the more difficult it is to obtain a numerical solution that is close to reality. On the other hand, although the simplification of the model makes it easier to solve the equations describing this model, the question arises whether the omitted impacts could not significantly influence the description of the phenomenon.

Very often, scientific papers uncritically apply various simplified models describing the phenomenon of water flow. In such cases, it seems reasonable to assess those which are applied most frequently. This paper compares three mathematical models describing the case of a two-dimensional low-velocity water flow through a straight 
channel sector with a cavern. For this purpose, a test stand allowing a water flow to be forced through a straight sector with a perpendicular lateral arm with no run-off (the so-called cavern) was constructed in the hydraulic laboratory at the Faculty of Civil and Environmental Engineering of the Gdańsk University of Technology (FCEE GUT). The research stand was built in a laboratory channel capable of ensuring an inflow and outflow of water of a given discharge and depth. Thanks to maintaining a proper depth, it was possible to measure velocity distribution with an ADV instrument (by SonTek) equipped with a "down-looking" probe, which measures three components of the velocity vector. The measurements were taken at selected locations. The measured values of two components of the velocity vector in the $x y$ horizontal plane (assuming average values in the vertical plane) were used for comparison with the results of numerical solutions.

\section{Mathematical Models}

In general, equations describing water movement are mathematical expressions of the laws of conservation of mass and momentum, complemented by the constitutive equation defining the relation between shear stress and viscosity. Let us assume that the flow is stationary, incompressible, isothermal and laminar, and the fluid examined is a Newtonian fluid. As a result of these assumptions, equations describing the velocity field and the flow pressure in the two-dimensional case will take the following forms:

$$
\begin{gathered}
\rho \cdot \boldsymbol{u} \cdot(\nabla \boldsymbol{u})=-\nabla p+\mu \cdot \Delta \boldsymbol{u}+\rho \cdot \boldsymbol{f}, \\
\nabla \cdot \boldsymbol{u}=0,
\end{gathered}
$$

where:

$$
\begin{aligned}
& \boldsymbol{u}-\text { velocity vector, } \boldsymbol{u}=\left[u_{x}, u_{y}\right] \\
& \boldsymbol{f}-\text { body force vector, } \boldsymbol{f}=\left[f_{x}, f_{y}\right] \\
& p-\text { pressure, } \\
& \mu-\text { dynamic viscosity coefficient }(\mu=\text { const }) \\
& \rho-\text { density of fluid, } \\
& \nabla-\text { differential operator nabla, }
\end{aligned}
$$

$$
\nabla=\frac{\partial}{\partial x} i+\frac{\partial}{\partial y} j
$$

$\Delta-$ Laplace differential operator,

$$
\Delta=\frac{\partial^{2}}{\partial x^{2}}+\frac{\partial^{2}}{\partial y^{2}}
$$

$\boldsymbol{i}, \boldsymbol{j}-\quad$ unit vectors of the coordinate system of spatial axes $x, y$. 
Equation (1) results from the principle of conservation of momentum, while equation (2) results from the principle of conservation of mass. The system of equations $(1,2)$ is known in the literature as the Navier-Stokes system of equations for an incompressible fluid in a stationary case (Landau and Lifshitz 1959). The Stokes model is the next model analysed in the current paper. It results directly from the Navier-Stokes equations, once we assume that we shall omit the influence of inertia forces in the description of the flow. In practice, this condition is determined with relation to the Reynolds number. After introducing the characteristic velocity $U^{*}$ and the characteristic linear dimension $L^{*}$, we can write a dimensionless version of equation (1) with reference to "modified" velocities $\boldsymbol{u}^{\prime}=\boldsymbol{u} / U^{*}$ and pressure $p^{\prime}=p L^{*} / \mu U^{*}$ :

$$
\operatorname{Re} \boldsymbol{u}^{\prime} \cdot\left(\nabla \boldsymbol{u}^{\prime}\right)=-\nabla p^{\prime}+\Delta \boldsymbol{u}^{\prime}+\frac{\operatorname{Re}}{\operatorname{Fr}} \cdot \frac{\boldsymbol{f}}{|\boldsymbol{f}|},
$$

where

$$
\begin{aligned}
& \operatorname{Re}-\text { Reynolds number: } \operatorname{Re}=\frac{U^{*} \cdot L^{*}}{v}, \\
& v-\text { kinematic viscosity } v=\frac{\mu}{\rho}, \\
& \text { Fr }- \text { Froude number: } \mathrm{Fr}=\frac{U^{* 2}}{|\boldsymbol{f}| \cdot L^{*}} .
\end{aligned}
$$

The Reynolds number is characterized by the relation of inertial forces to viscous forces, whereas the Froude number describes the ratio of inertial forces to gravity. Therefore, if we assume that inertial forces are low in comparison to viscous forces (a low Re value), the left side of equation (5) may be omitted. Returning to the dimensional variables, equation (1) changes to

$$
-\nabla p+\mu \Delta \boldsymbol{u}+\rho \cdot \boldsymbol{f}=0 .
$$

The system of equations $(6,2)$ describes Stokes flow. It may be noticed that, unlike equation (1), equation (6) is linear, which greatly facilitates the process of numerical solving. The third model to be analysed is the two-dimensional potential flow model. In this specific case, we assume that the velocity vector has the potential $\varphi$, which means that $\boldsymbol{u}=\operatorname{grad}(\varphi)$, and that the fluid is inviscid or the viscous forces do not have any significant influence on the character of the flow. Therefore, the system of equations $(1,2)$ is transformed to a harmonic equation with relation to the function $\varphi$ :

$$
\Delta \varphi=0 .
$$

The equations presented above, defining particular mathematical models of the flow, belong to the category of partial differential equations (PDE), which require the correlation of some additional data in order to be solved (LeVeque 1990, Tannehill et al 1997). These additional pieces of information include a description of the domain 
where the solution and its proprieties are defined. The boundary conditions complete the process of formulating the problem. In the case of equations describing stationary processes, we usually have to deal with the so-called boundary problems.

\section{Numerical Solution}

The next step is to obtain solutions of these problems. Over the last hundred years, many different methods of approximate solution of partial differential equations have been developed (Hirsch 1990). The most common are the finite-difference method (FDM), the finite element method (FEM), the finite-volume method (FVM) and the boundary element method (BEM) (Cheng and Cheng 2005). While the first three methods are based on formulating the problem through partial differential equations, the last one requires problem formulation in the form of integral equations which are derived from differential equations. And if, for the first group of methods, the discretization of the whole domain is obligatory (since unknown functions are defined in the whole domain), in the case of BEM, everything depends on a particular integral equation. Very often, this equation contains unknown functions defined only on the boundary of the domain, which results in reducing the process of discretization to its boundary only.

The major advantages of BEM are, above all, a significant facilitation of the discretization process (in comparison to ordinary mesh methods), a reduction of the number of discrete elements and the computation time, and high efficiency in the case of problems formulated in infinite domains (Banerjee, Butterfield 1981). In the present study, we used the BEM method to solve the system of equations $(6,2)$ and the harmonic equation (7). However, we first had to rearrange these equations to an integral form. Later in the paper, we will present this process for Stokes equations. In the case of the harmonic equation, we will refer to integrate equations (without actually introducing them), which are known from classical potential theory.

In order to solve the system of equations $(1,2)$, we used OpenFOAM (Open source Field Operation And Manipulation), a program with an open source code based on FVM. In FVM (LeVeque 2002), these equations are integrated in areas surrounding the nodes of the calculation grid. Double integrals containing divergent terms are transformed into surface integrals using the divergence theorem. This approach allows existing characteristics to be considered as streams across the surface of each finite volume. Such a formulation leads to a "natural" fulfillment of the condition of maintaining a size of the flux passing through the border of neighboring grid elements. This means that in the case of equations $(6,2)$, equation (2) is automatically satisfied. The Pressure-Implicit with Splitting of Operators (PISO) algorithm (Issa 1985) is used to solve equations $(1,2)$ in OpenFOAM.

With regard to boundary conditions, it should be noted that in equations $(1,2)$ and $(6,2)$, for each point belonging to the boundary of the domain, it is necessary to give a flow velocity vector or a stress vector - or mixed conditions when the boundary 
condition vector is composed of the velocity vector and the stress vector. There are two types of boundary conditions for equation (7): Dirichlet conditions ( $\varphi$ value is given) and Neumann conditions ( $\boldsymbol{u}_{n}$ velocity in the direction normal to the boundary is given). Further in the current paper, we will analyse only the boundary conditions referring to velocity.

\section{Integral Presentation of Two-dimensional Stokes Equations}

As mentioned above, in order to solve equations $(6,2)$, BEM was applied. At the very beginning, it is necessary to transform the equations into their integral forms. The transformation of the integral equations corresponding to $(6,2)$ is described in detail in the papers (Pozrikidis 1992, Hahn 2013). For this reason, in the following part of this article, only the major aspects of this transformation are shown.

First, we write the Lorentz principle of reciprocal identity for Stokes flow (Pozrikidis 1992). Let us assume that $\boldsymbol{u}$ and $\boldsymbol{u}^{*}$ are two solutions of the Stokes flow equations with their respective stress tensors $\sigma$ and $\sigma^{*}$. Then, taking into consideration relation (2), we can write

$$
\nabla \cdot\left(\boldsymbol{u}^{*} \cdot \boldsymbol{\sigma}-\boldsymbol{u} \cdot \boldsymbol{\sigma}^{*}\right)=0
$$

where $\boldsymbol{u}^{*}, \boldsymbol{\sigma}^{*}$ and $p^{*}$ are, respectively, flow, stress and pressure fields induced by the point force $\boldsymbol{f}$ located at the point $\boldsymbol{x}_{0}$ (also known as the fundamental solution of Stokes equations, or Green's functions for two-dimensional Stokes equations). Using the approach presented in the papers (Lisicki 2013, Pozrikidis 1992), we can write definitions of individual terms:

$$
\begin{gathered}
u_{i}^{*}(\boldsymbol{x})=\frac{1}{4 \pi \mu} G_{i j}(\boldsymbol{r}) f_{j}, \\
\sigma_{i k}^{*}(\boldsymbol{x})=\frac{1}{4 \pi} T_{i j k}(\boldsymbol{r}) f_{j}, \\
p^{*}(\boldsymbol{x})=\frac{1}{4 \pi} P_{j}(\boldsymbol{r}) f_{j},
\end{gathered}
$$

where

$$
\begin{gathered}
G_{i j}=-\delta_{i j} \ln |\boldsymbol{r}|+\frac{r_{i} r_{j}}{|\boldsymbol{r}|^{2}}, \\
P_{j}=2 \frac{r_{j}}{|\boldsymbol{r}|^{2}}, \\
T_{i j k}=-4 \frac{r_{i} r_{j} r_{k}}{|\boldsymbol{r}|^{4}},
\end{gathered}
$$

$f_{j}$ - component of the mass force vector,

$i, j, k=1,2$, 
$\boldsymbol{r}-\boldsymbol{x}_{0}-\boldsymbol{x}$,

$\boldsymbol{x}, \boldsymbol{x}_{0}-$ any points in a two-dimensional space,

$\delta-$ Kronecker delta

$$
\delta=\left\{\begin{array}{l}
0, i \neq j \\
1, i=j
\end{array}\right.
$$

The tensor $T$, in particular, is defined as

$$
T_{i j k}(\boldsymbol{r})=-\delta_{i k} P_{j}(\boldsymbol{r})+\frac{\partial G_{i j}}{\partial x_{k}}(\boldsymbol{r})+\frac{\partial G_{k j}}{\partial x_{i}}(\boldsymbol{r}) .
$$

Integrating (8) over the area $S$, we obtain the following:

$$
\iint_{S} \frac{\partial}{\partial x_{k}}\left[G_{i j}(\boldsymbol{r}) \cdot \sigma_{i k}-\mu u_{i} \cdot T_{i j k}(\boldsymbol{r})\right] d S=0 .
$$

Taking into account the singularity $G(\boldsymbol{r}), T(\boldsymbol{r})$ for the case $\boldsymbol{x} \rightarrow \boldsymbol{x}_{0}$, when $\boldsymbol{x}_{0}$ is placed inside or on the border of the area $S$, applying the divergence theorem and introducing the surface stress vector $\boldsymbol{F} \boldsymbol{s}=\boldsymbol{\sigma} \cdot \boldsymbol{n}$, we obtain (Pozrikidis 1992)

$$
w\left(\boldsymbol{x}_{0}\right) \cdot \boldsymbol{u}\left(\boldsymbol{x}_{0}\right)=\frac{1}{\mu} \oint_{\Omega} \boldsymbol{F} \boldsymbol{s}(\boldsymbol{x}) \cdot G(\boldsymbol{r}) d \Omega+\mu \oint_{\Omega} \boldsymbol{u} \cdot T(\boldsymbol{r}) \cdot \boldsymbol{n}(\boldsymbol{x}) d \Omega,
$$

where $\boldsymbol{x}$ is now placed on the boundary $\Omega$,

$\Omega \quad-$ boundary of the domain $S(\Omega \not \subset S)$,

$\boldsymbol{n}(\boldsymbol{x}) \quad-$ external vector normal to $\Omega$ at the point $\boldsymbol{x}$,

$$
w\left(\boldsymbol{x}_{0}\right)-\text { weighting coefficient }\left\{\begin{array}{c}
4 \pi, \boldsymbol{x}_{0} \in S \\
2 \pi, \boldsymbol{x}_{0} \in \Omega \\
0, \boldsymbol{x}_{0} \notin \Omega \wedge \boldsymbol{x} \notin S
\end{array} .\right.
$$

It must be pointed out that equation (18) directly allows the boundary conditions to be given in the form of the stress vector only or in the form of the velocity vector only or as mixed conditions of these two types. However, we should not forget that we assumed the boundary conditions in the form of the velocity vector. In such a case, when giving the boundary conditions, we have to remember to maintain an identical balance of water flowing into and out of the solution domain (satisfying the conservation of mass equation).

\section{Integral Presentation of Two-dimensional Laplace Equations}

Using classical potential theory for Laplace's equation, we can write the so-called single layer potential (Brebbia et al 1984) of the function $\varphi$ :

$$
\varphi\left(\boldsymbol{x}_{0}\right)=\oint_{\Omega} \omega(\boldsymbol{x}) \cdot \varphi^{*}(\boldsymbol{r}) \cdot d \Omega,
$$


where:

$$
\begin{aligned}
& \Omega \quad-\text { boundary of the domain } S(\Omega \not \subset S), \\
& \varphi^{*}(\boldsymbol{r})-\quad \text { fundamental solution of Laplace's equation for a two-dimensional } \\
& \quad \text { case } \varphi^{*}=\ln (1 /|\boldsymbol{r}|), \\
& \omega(\boldsymbol{x})-\quad \text { unknown function, a surface density of the velocity potential. }
\end{aligned}
$$

To apply the Neumann condition, let us consider the extreme situation when the point $\boldsymbol{x}_{0}$ lies on the edge of the area $\left(\boldsymbol{x}_{0} \rightarrow \boldsymbol{x}\right)$. The derivative of the left and right side of equation (19) in the direction (x) normal to $\Omega$ will be equal to

$$
\lim _{\boldsymbol{x}_{0} \rightarrow \boldsymbol{x}} \frac{\partial}{\partial \boldsymbol{n}\left(\boldsymbol{x}_{0}\right)} \varphi\left(\boldsymbol{x}_{0}\right)=\lim _{\boldsymbol{x}_{0} \rightarrow \boldsymbol{x}} \oint_{\Omega} \omega(\boldsymbol{x}) \cdot \frac{\partial}{\partial \boldsymbol{n}\left(\boldsymbol{x}_{0}\right)} \varphi^{*}(\boldsymbol{r}) \cdot d \Omega .
$$

Taking into account the singularity of the sub-integral expression in the right side of (20), calculating the limits, we will obtain (Brebbia et al 1984)

$$
u_{n}\left(\boldsymbol{x}_{0}\right)=-\pi \omega(\boldsymbol{x})+\oint_{\Omega} \omega(\boldsymbol{x}) \cdot \frac{\partial \varphi^{*}(\boldsymbol{r})}{\partial \boldsymbol{x}(x)} \cdot d \Omega,
$$

where $\boldsymbol{x}_{0}$ is now placed on the boundary $\Omega$.

Equation (21) is a Fredholm equation of the second kind. Using the boundary condition of the Neumann type, the function $\omega(\boldsymbol{x})$ is determined from equation (21). By substituting the omega function for equation (19) we obtain the expression for the potential at each point of the area $S$.

\section{Laboratory Tests}

In order to proceed with the validation analysis within the scope of the current paper, we chose the case of a two-dimensional flow through a straight channel sector with a no-run-off arm in the form of a rectangular cavern. A $62 \mathrm{~cm}$-wide test stand was constructed in a laboratory flume at the hydraulic laboratory of FCEE GUT. The channel is supplied with water pumped in from the laboratory's closed cycle. The pumps are equipped with frequency converters making it possible to set precisely the intensity of water inflow into the channel, as well as maintaining fixed conditions. A straight channel sector with a rectangular cavern was constructed of small cement blocks placed in the straight sector of the laboratory channel with a horizontal cement bottom (Fig. 1).

In the structure, we marked points where velocity measurements were taken (indicated with red crosses in Fig. 1). The single-point velocity was measured with an ADV instrument (by SonTek) equipped with a "down-looking" probe and capable of measuring three components of the velocity vector. The ADV instrument was assembled on a special trolley over the channel so that it could be moved parallel and perpendicular to the channel's axis, as well as to locate the measurement cell at an 


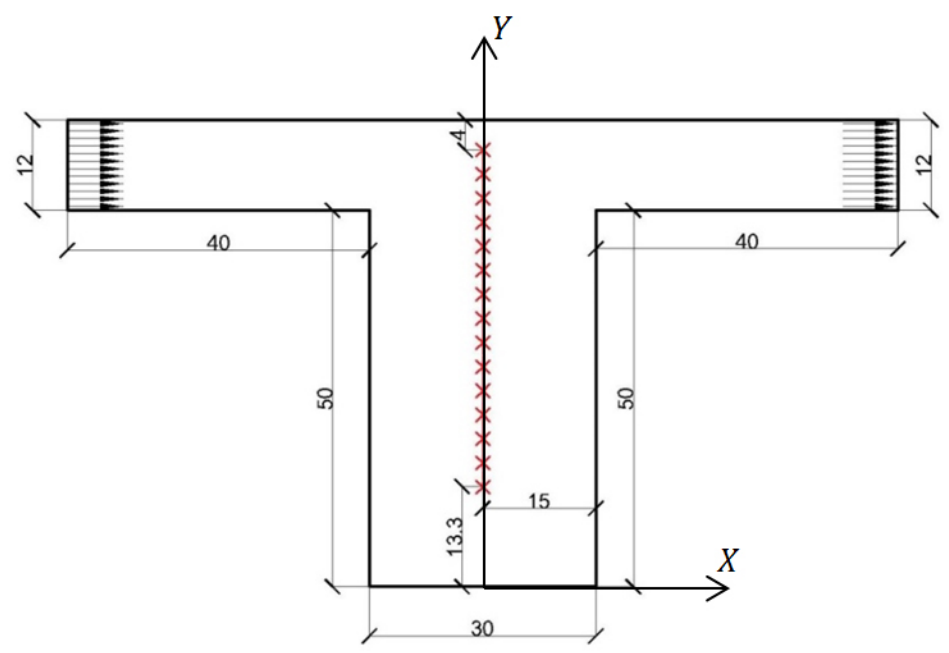

Fig. 1. The outline (horizontal view) of the cavern. The measurement points are marked with crosses. All values are expressed in $\mathrm{cm}$

appropriate distance from the bottom and lateral walls of the structure (about $15 \mathrm{~cm}$ from the bottom of the channel).

All conditions for the use of the ADV probe were fulfilled (the presence of particulates in the water). The placement of the investigated structure in the laboratory channel made it possible to impose suitable water flow conditions (a low velocity and an appropriate depth for measurements to be taken with the ADV instrument). The depth was maintained at $30 \mathrm{~cm}$ by damming water at the outlet of the channel. Several measurement series were conducted, each corresponding to a different inlet velocity: $0.3,1.0,3.0$ and $10.0 \mathrm{~cm} / \mathrm{s}$, achieved by controlling the water inflow into the duct. During the experiment, the probe of the ADV instrument was moved in the direction normal to the channel's axis, while the components of the velocity vector were measured at selected points (Fig. 1). At each measurement point, we recorded about 2000 measurements of each velocity vector component, and then we averaged all these values.

Fig. 2 presents the measurement results in a graphic form. It shows the averaged values of $V_{x}$ velocity component (the black points): they are correlated with the line of approximation (the green solid line), which approximates selected points along the middle of the cavern, and different values of the velocity $V_{i n}$ at the inlet to the straight sector preceding the cavern. A good practice in performing measurements of all kinds is to determine the measuring error (or the measurement uncertainty). One of the sensor's characteristics (Table 1) is the permanent measuring error $\Delta=2.5 \mathrm{~mm} / \mathrm{s}$, which limits the minimum velocity at which the results will not be burdened by excessive measuring errors. This means that the highest value of any relative measurement uncertainty $( \pm \Delta)$ for the components of the velocity vector is achieved at around zero. 
Another factor which influences the quality of measurement results is the presence of particulates, which allow measurements to be obtained on the basis of the Doppler effect. In simplified terms: the clearer the water, the bigger the measuring error of the ADV sensor. This results from the way the measurements are performed by the ADV method, which consists in "reflecting" the sound signal from elementary particles present in water.

a) $V_{\text {in }}=0.3 \mathrm{~cm} / \mathrm{s}$

$V_{x}(\mathrm{~cm} / \mathrm{s})$

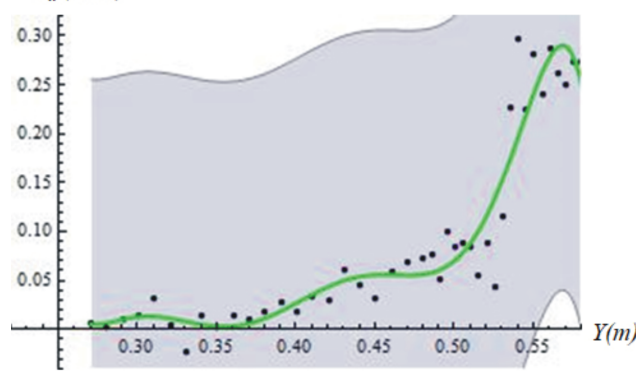

c) $V_{\text {in }}=3 \mathrm{~cm} / \mathrm{s}$

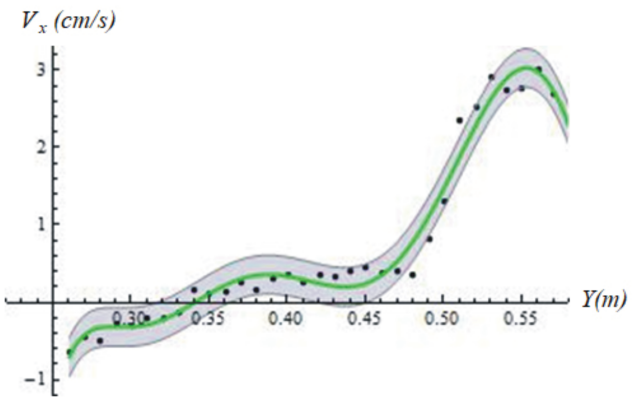

b) $V_{\text {in }}=1 \mathrm{~cm} / \mathrm{s}$

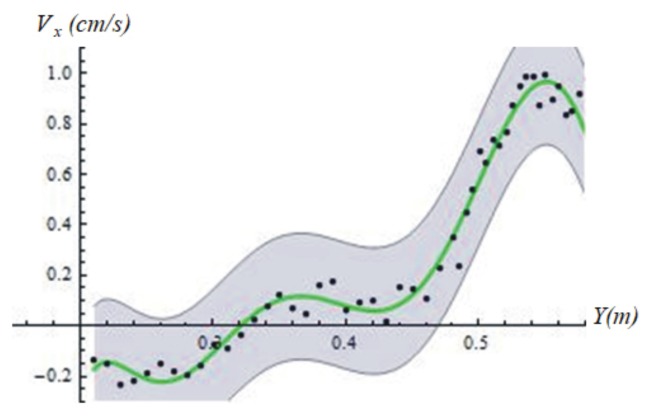

d) $V_{\text {in }}=10 \mathrm{~cm} / \mathrm{s}$

$V_{x}(\mathrm{~cm} / \mathrm{s})$

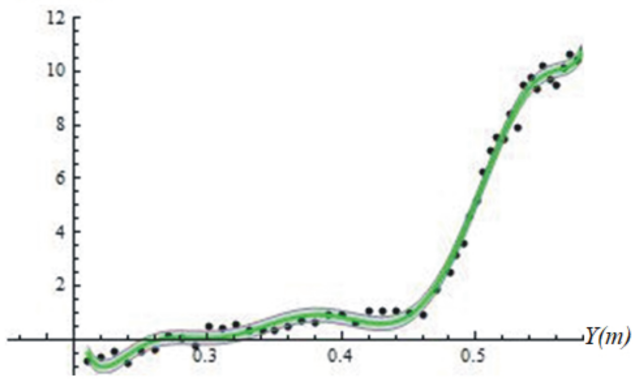

Fig. 2. The averaged values of the measured component of $V_{s}$ velocity for selected points. The green solid line approximates the results obtained, and the upper and lower bounds of the measuring error of the ADV instrument are shown

Table 1. Basic characteristics of the ADV sensor by SonTek

\begin{tabular}{|l|l|}
\hline measurement frequency & $0.1-50 \mathrm{~Hz}$ \\
\hline range of velocity measured & $1 \mathrm{~mm} / \mathrm{s}-2.5 \mathrm{~m} / \mathrm{s}$ \\
\hline measuring error & $2.5 \mathrm{~mm} / \mathrm{s}$ \\
\hline resolution & $0.1 \mathrm{~mm} / \mathrm{s}$ \\
\hline measurement cell & $0.09 \mathrm{~cm}^{3}$ \\
\hline measurement point distance & $5 \mathrm{~cm}$ \\
\hline
\end{tabular}

The measurement results were used to validate the chosen mathematical models describing water flow. 


\section{Numerical Tests}

For the purposes of this paper, we also conducted numerical tests to compare the adopted flow models, as well as to show their characteristic properties. These properties are related to the assumptions underlying each model. As has already been noted, in order to conduct a comparative analysis, we chose the case of a two-dimensional flow through a straight channel sector with a rectangular cavern. This case allows us to clearly reveal differences between the solutions obtained, depending on which of the flow models was adopted. Moreover, it is frequently used to verify accepted solutions in CFD (Erturk et al 2005, Zienkiewicz and Taylor 2000). During the tests, the flow through a straight sector of the flume with a cavern was reconstructed (Fig. 1).

a) $\operatorname{Re}=960$

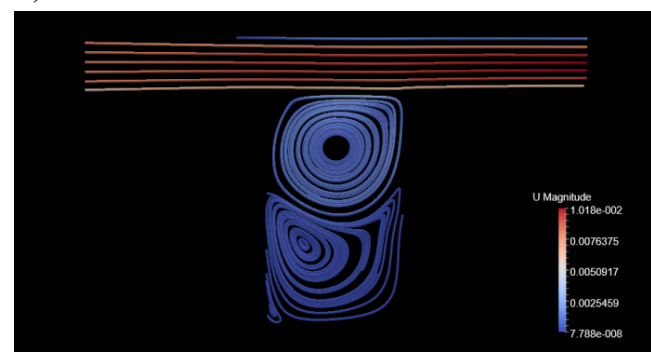

c) $R e=9.6$

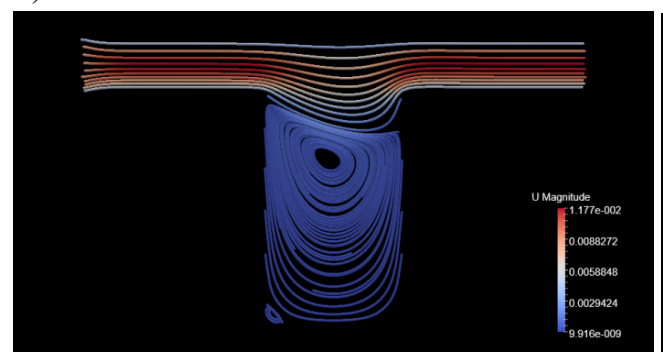

b) $R e=96$

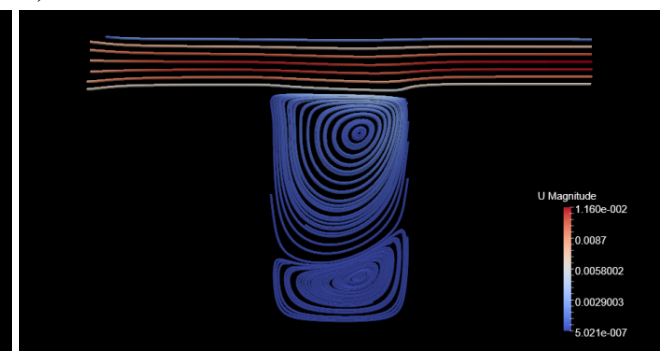

d) $R e=0.96$

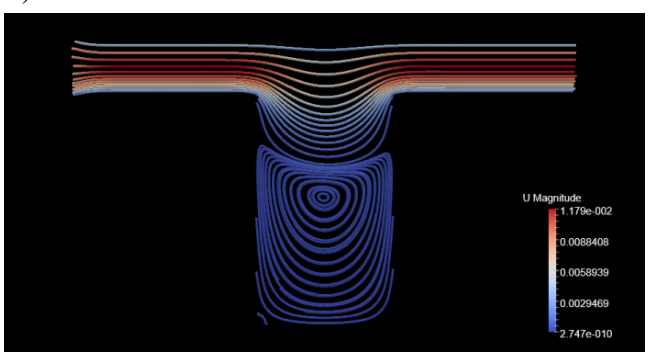

Fig. 3. Trajectories of fluid particles calculated on the basis of the Navier-Stokes model for different Reynolds numbers by the OpenFOAM program (FVM applied)

Fig. 3 presents the results of water flow through a straight flume with a cavern modelled in the OpenFOAM program (the solution obtained by FVM) in the form of streamlines (tangent to the velocity vector, overlapping the trajectory of particles in a stationary motion). The domain was divided by means of a homogeneous mesh consisting of 45120 cubes (the OpenFOAM program was designed to model 3D problems). We can clearly see that, regardless of the hydraulic parameters of the flow, a closed vortex structure develops inside the cavern. It is tangent to the rectilinear flow along the cavern's straight sector. The results are presented for different values of the Reynolds number (Fig. 3a-d). Let us point out that the trajectory position of particles depends on the value of the Reynolds number (between $\operatorname{Re} \approx 960$ and $\operatorname{Re} \approx 0.96$ ). 
Below $\operatorname{Re}=1$, a decrease in its value has no significant impact on the character of the flow.

Fig. 4(a-b) show streamlines calculated by the simplified models: the Stokes flow model (Fig. 4a) and the two-dimensional potential flow model (Fig. 4b). In both cases, BEM was applied to solve them. During domain discretization in accordance with BEM, 3440 nodes were obtained.

a)

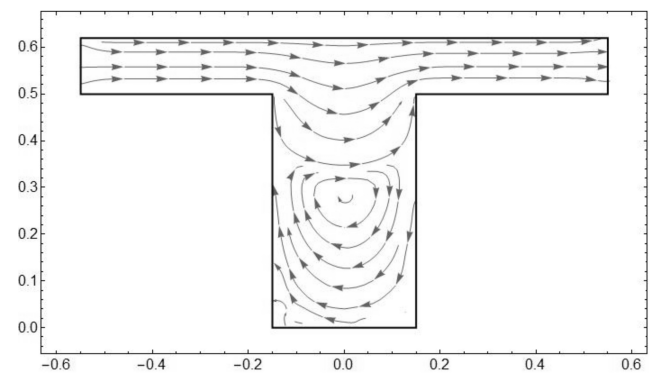

b)

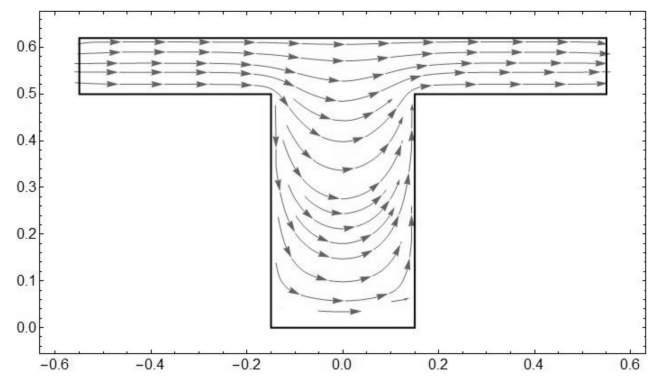

Fig. 4. a) Flow streamlines in the cavern calculated on the basis of the Stokes model; b) Flow streamlines in the cavern calculated on the basis of the two-dimensional potential flow model

The results obtained from the Stokes model and from the two-dimensional potential flow model are different from those obtained on the basis of the Navier-Stokes model. First of all, the shape and position of streamlines do not change depending on the flow parameters. This results from the fact that in the former two models, we omit the influence of inertial forces. The Stokes model isolates a closed vortex structure tangent to the flow along the cavern's straight sector. This structure is symmetrical and independent of the flow velocity. The two-dimensional potential flow model forecasts the total absence of fluid particle rotation during its movement, leading to the omission of the influence of viscosity forces. As a result, there are no visible closed vortex structures. The streamlines, regardless of the cavern's size, lie parallel to one another.

\section{Comparison of the Results of Numerical Simulations with the Results of Measurements}

In order to proceed with the comparison, we juxtaposed the results (of calculations and measurements) on the line marked with red crosses in Fig. 1. The section thus prepared allowed us to compare the velocity component $V_{x}$ as an important value of the velocity vector component. Since the initial analysis revealed that the results from the two-dimensional potential flow model differed significantly from the actual character of the flow (as well as from the results obtained by the remaining two models), it was not ultimately used in the current comparison. 
Subsequently, a flow with an inlet horizontal velocity of around $0.8 \mathrm{~cm} / \mathrm{s}$ was chosen. In this case, the Reynolds number equals about 960, which means that, in reality, the flow has to be turbulent. Fig. 5 compares the results obtained. It is possible to notice that in the case of a decreasing Reynolds number, the curves corresponding to the Navier-Stokes model approach the curve calculated on the basis of the Stokes model. The Navier-Stokes model yields relatively good results in modelling flows close to laminar flows. Moreover, it is worth mentioning that, although the green curve (corresponding to the Stokes model) lies relatively far from the points depicting the measurement results, it allows a "general" approximate shape of the velocity profile to be determined.

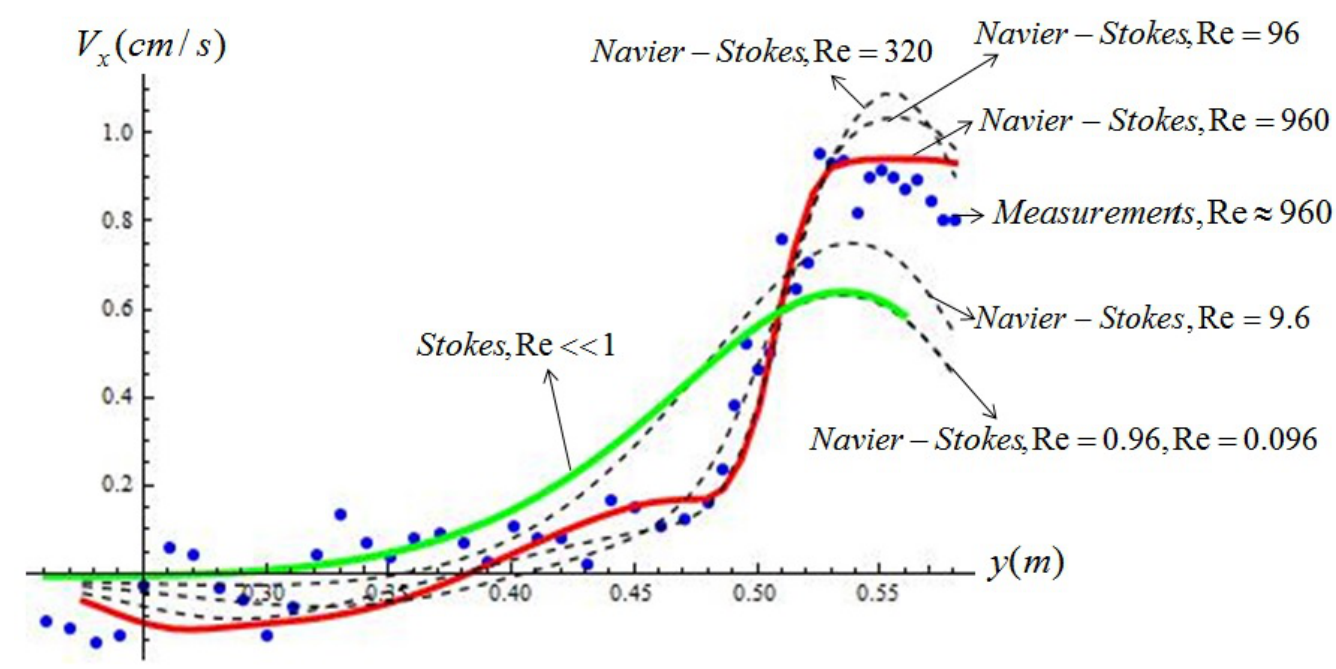

Fig. 5. The velocity component $V_{x}$ calculated on the basis of the Stokes flow model and the Navier-Stokes model (for different Reynolds numbers) as well as the averaged values of the velocity component $V_{x}$ measured in the laboratory

\section{Conclusions}

The results of the research allow us to conclude that the simplified models used in describing water flows may also be used to model certain classes of real problems. Their specific properties significantly influence the solutions obtained and may limit their usefulness in particular cases. For instance, in modelling water flow in a two-dimensional cavern, the two-dimensional potential flow model is inaccurate to the extent that it does not even allow the general shape of the velocity domain to be visualized. The Stokes flow model, on the other hand, enables us to visualize it and, what is also important, to find the zone of a no-run-off water vortex - a vortex in the lower part of the cavern. It may be stated that in some cases (cases of a flow 
with a low Reynolds number, in particular), this model can be applied to achieve the adopted modelling objectives (such as the location of closed vortex zones).

In numerical tests, the best results were obtained by the Navier-Stokes model. However, since the application of this particular model requires solution of nonlinear equations, it may cause some issues in numerical solving. In conclusion, the application of a particular simplified model should be carefully thought out, and its features should be compared with the features of the modelled object.

\section{References}

Andrews J. M., McLone R. R. (1976) Mathematical modeling, London, Butterworths.

Banerjee P. K., Butterfield R. (1981) Boundary Element Methods in Engineering Science, McGraw-Hill, London, New York.

Brebbia C. A., Telles J. C. F., Wrobel L. C. (1984) Boundary Element Techniques. Theory and Application in Engineering, Springer-Verlag, Berlin. Heidelberg, New York, Tokio.

Cheng A., Cheng D. (2005) Heritage and early history of the boundary element method, Engineering Analysis with Boundary Elements, 29, brak numerów stron, jeśli to czasopismo.

Erturk E., Corke T. C., Gokcol C. (2005) Numerical Solutions of 2-D Steady Incompressible Driven Cavity Flow at High Reynolds Numbers, International Journal for Numerical Methods in Fluids, 48 (7), 747-774.

Hahn M. (2013) Master's thesis: Investigation of the boundary integral method for Stokes flow, University of Bayreuth, Bayreuth.

Hirsch C. (1990) Numerical Computation of Internal and External Flows, volume 2: Computational Methods for Inviscid and Viscous Flows, Wiley, Hoboken.

Issa R. I. (1985) Solution of the Implicitly Discretized Fluid Flow Equations by Operator-Splitting, Journal of Computational Physics, 62, 40-65.

Landau L. D., Lifshitz E. M. (1959) Fluid Mechanics (Volume 6 of A Course of Theoretical Physics), Pergamon Press.

LeVeque R. (1990) Numerical Methods for Conservation Laws, ETH Lectures in Mathematics Series, Birkhauser-Verlag.

LeVeque R. (2002) Finite Volume Methods for Hyperbolic Problems, Cambridge University Press.

Lisicki M. (2013) Four approaches to hydrodynamic Green's functions - the Oseen tensors, Institute of Theoretical Physics, Faculty of Physics, University of Warsaw.

Meerschaert M. M. (2007) Mathematical Modeling, 3rd ed., Elservier Science.

Pozrikidis C. (1992) Boundary Integral and Singularity Methods for Linearized Viscous Flow, Cambridge University Press, Peter Phillips, Cambridge.

Tannehill J. C. et al (1997) Computational Fluid Mechanics and Heat Transfer, 2nd ed., Taylor and Francis.

Zienkiewicz O. C., Taylor R. L. (2000) The Finite Element Method, Vol. 3. Fluid Dynamics, Butterworth-Heinemann. 